\title{
Glycoconjugates play a key role in Campylobacter jejuni infection: interactions between host and pathogen
}

\section{Christopher James Day ${ }^{\dagger}$, Evgeny Alexander Semchenko ${ }^{\dagger}$ and Victoria Korolik*}

Institute for Glycomics, Griffith University, Gold Coast, OLD, Australia

Edited by:

Alain Stintzi, Ottawa Institute of

Systems Biology, Canada

\section{Reviewed by:}

Marina Šanti, University of Rijeka,

Croatia

Christine M. Szymanski, University of Alberta, Canada

\section{*Correspondence:}

Victoria Korolik, Institute for Glycomics, Griffith University, Gold

Coast, QLD 4222, Australia. e-mail:v.korolik@griffith.edu.au

${ }^{+}$Christopher James Day and Evgeny Alexander Semchenko have contributed equally to this work.
Glycan based interactions between host and pathogen are critical in many bacterial and viral diseases. Glycan interactions range from initial receptor based adherence to protecting the infective agent from the host's immune response through molecular mimicry. Campylobacter jejuni is an ideal model for studying the role of glycans in host-pathogen interactions, as well as the role of bacterial surface glycoconjugates in infection. Using glycan array analysis, $C$. jejuni has been shown to interact with a wide range of host glycoconjugates. Mannose and sialic acid residues appear to play a role in initial interactions between host and pathogen following environmental exposure, whereas fucose and galactose based interactions are likely to be required for prolonged colonization. Other studies have highlighted potential decoy receptor type interactions between host's intestinal mucins and $C$. jejuni, demonstrating the importance of host glycoproteins as defense against $C$. jejuni infection as well as the role for glycoconjugates found in human breast milk in protection of breast feeding infants from infection with $C$. jejuni. C. jejuni can produce $\mathrm{N}$ - and $\mathrm{O}$ linked glycoproteins, capsular polysaccharide (CPS) and/or lipooligosaccharide (LOS) which results in $C$. jejuni presenting its own diverse sugar coated displays on the cell surface. Bacterial glycans play an important and versatile role in infection and disease. Of these, the best understood is the molecular mimicry of human gangliosides presented by $C$. jejuni's LOS and its link to the onset of autoimmune neuropathies such as the Guillain Barrè syndrome (GBS). However, the role of glycoconjugates presented by $C$. jejuni extends beyond expression of sialylated ganglioside structures involved in initiation of GBS. Expression of surface glycans by $C$. jejuni may also relate to the ability of this organism to interact with the glycoproteins for initial host-pathogen interactions and continued infectivity.

Keywords: glycans, LOS, glycoconjugates

\section{INTRODUCTION}

Glycosylation is a ubiquitous decoration of proteins, lipids, and other molecules (such as organic acids) with carbohydrate structures by all living cells (Lehmann et al., 2006). For pathogenic bacteria both the presentation of surface glycans and the recognition of host cell glycans are crucial for the survival in, colonization, and continued infection of host tissues (Lehmann et al., 2006). Campylobacter jejuni has been shown to present a wide array of glycans to aid in the evasion of the host immune responses and to protect itself from host defenses, including proteolytic degradation (Karlyshev et al., 2005b). Recently C. jejuni has also been shown to bind to a diverse range of host glycans that are potentially crucial for the initial attachment to and continued colonization of the host (Morrow et al., 2005; Day et al., 2009). Here we discuss recent data on both, the factors involved in the heterogeneous expression of lipooligosaccharide (LOS) by C. jejuni and the importance of the interactions of $C$. jejuni with host glycans for colonization and infection.

\section{IMPORTANCE OF GLYCANS FOR INFECTION}

To infect a host, bacterial pathogens must be equipped with multiple factors to assist in colonization and depending on the site of infection, these factors vary widely. Some bacterial species must be highly motile and sensitive to small chemical gradients to successfully locate their niche, while other species require no self motility to successfully infect the host tissues they prefer. Initial contact between host and pathogen occurs most frequently through bacterial surface components that mediate adherence which are collectively called adhesins. As the host cell surfaces display multitude of glycoconjugates, it is not surprising that a large number of bacteria express carbohydrate specific adhesins and that expression of specific lectin style adhesins is thought to be responsible for the tissue tropism of pathogens (Sokurenko et al., 1998; Lehmann et al., 2006; Lloyd et al., 2007).

A diverse range of glycoconjugates has now been identified as targets of pathogenic microbes including fucosylated glycans (e.g., Pseudomonas aeruginosa), gangliosides, and lactoceramides (e.g., Helicobacter pylori), sialylated glycans (e.g., Streptococcus gordonii), and mannosylated structures (e.g., Escherichia coli; GilboaGarber et al., 1994; Thomas et al., 2002; Lehmann et al., 2006; Yajima et al., 2008). One of the best defined glycan dependent interactions with host tissues is the recognition of mannosylated glycoconjugates by the FimH protein of E. coli (Sokurenko et al., 1994, 1998; Klemm et al., 1996; Aprikian et al., 2007; Rosen et al., 
2008; Sepehri et al., 2009). Not only has this been defined as a key interaction required for colonization, commensalism, and infections with E. coli; but also depending on the chain length of the mannose recognized by FimH, it identifies the favored niche of the E. coli strain (Sokurenko et al., 1998; Pouttu et al., 1999; Rosen et al., 2008). E. coli strains that interact through FimH with short repeat mannosylated glycoconjugates tend to be uropathogenic, while those that recognize longer mannose repeats tend to be found as either commensals or pathogens in intestinal and colonic tissues (Sokurenko et al., 1998; Rosen et al., 2008). Many other bacteria, including Streptococcus spp. and Neisseria spp., have been shown to require interactions with host glycans for successful infection (Lehmann et al., 2006; Lloyd et al., 2007; Sharon, 2008), however, the factors involved in these interactions have not always been elucidated (Scharfman et al., 1999; Day et al., 2009).

\section{DEFINED GLYCAN BINDING IN THE EPSILONPROTEOBACTERIA: HELICOBACTER PYLORI}

Carbohydrate based interactions have not been fully solved for $C$. jejuni, but the related species Helicobacter pylori has been extensively studied and the lectins involved in carbohydrate dependent interactions have been well characterized (Utt and Wadstrom, 1997; Ilver et al., 1998; Mahdavi et al., 2002; Cooksley et al., 2003; Hynes et al., 2003; Bennett and Roberts, 2005; Walz et al., 2005). H. pylori exhibits broad complexity in carbohydrate-binding specificity, owing to the expression of six different lectins/adhesins with specificities for sialylated oligosaccharides, Lewis B antigen, lactosylceramide and ganglioseries glycolipids, and heparan sulfate (Utt and Wadstrom, 1997; Ilver et al., 1998; Mahdavi et al., 2002; Cooksley et al., 2003; Hynes et al., 2003; Bennett and Roberts, 2005; Walz et al., 2005). Of these six lectins, only four (SabA, BabA, HapA, and $\mathrm{Hbp}$ ) are actually involved in adherence of the bacteria to host tissues, while the other two (HP-NAP and HP0721) appear to be involved in the "mopping up" of free sialic acid in chronically infected tissue. It has been proposed for H. pylori that initial interactions with host tissues may be achieved through binding to non-sialylated glycoconjugates present in the normal gastric epithelium (e.g., Lewis B antigen and glycosphingolipids). While, the sialic acid binding capacity of $H$. pylori is likely to enable persistence of $H$. pylori infection by mediating adhesion through the sialic acid binding lectin, SabA, to the already diseased epithelium of the stomach of chronically infected hosts (Figure 1; Mahdavi et al., 2002).

\section{CAMPYLOBACTER JEJUNI GLYCAN DEPENDENT INTERACTIONS WITH HOST TISSUES}

Like H. pylori, C. jejuni has been reported to have a broad binding specificity for glycans and is known to interact with mucins and other glycoproteins (Hugdahl et al., 1988; McAuley et al., 2007; Tu et al., 2008; Stahl et al., 2011). Through glycan array based analysis, we have identified the binding of C. jejuni strain NCTC11168 to galactose, fucose, sialic acid, mannose, glucosamine, and glycosaminoglycans, however, the interactions with glycans were dependent on the conditions that $C$. jejuni were grown or maintained (Day et al., 2009). C. jejuni NCTC11168 was found to bind glycans terminating in galactose regardless of linkage (both $\alpha$ -

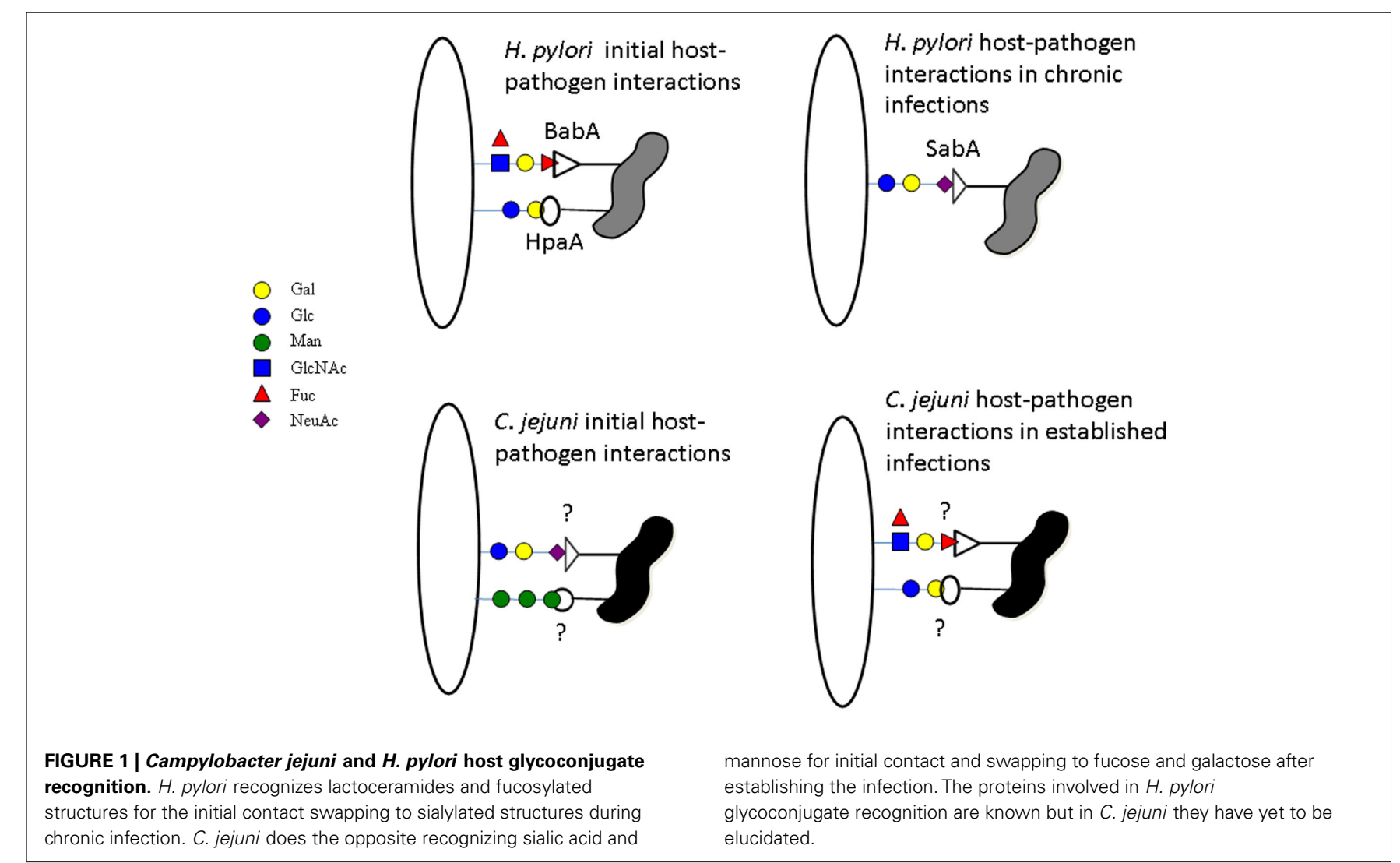


and $\beta$-linked) and fucosylated glycans when grown under conditions mimicking mammalian and avian hosts. Less binding was observed to fucosylated and terminal galactose structures when maintained in conditions mimicking environmental stress (Day et al., 2009). Conversely, direct interactions between C. jejuni NCTC11168 and sialic acid or mannose containing glycoconjugates was observed in glycan array experiments but only after the bacteria were put under environmental stress (Day et al., 2009). The glycan based interactions observed for C. jejuni NCTC11168 under the different conditions has been replicated in numerous other strains (unpublished data). Of the 11 other $C$. jejuni strains [human isolates: $11168 ; 351 ; 375 ; 520 ; 81116$ and $81-176$ and chicken isolates: $008 ; 019 ; 108 ; 331 ; 434$ and 506$]$ tested so far at all three conditions (environmental, 37 , and $42^{\circ} \mathrm{C}$ )], nine have been found to bind to almost identical structures under the same conditions as C. jejuni NCTC11168. The two exceptions are atypical C. jejuni strains, still under investigation. These glycan dependent interactions and the way in which $C$. jejuni is able to modulate its binding of glycoconjugates under different conditions, allows us to hypothesize that mannose and sialic acid based adherence is required for initial contact with host tissues after exposure to the external environment, with long term infection resulting from interactions with fucose and terminal galactose containing glycoconjugates (Figure 1; Day et al., 2009).

It is interesting to note the differences in the way that C. jejuni and $H$. pylori interact with glycoconjugates may explain why bacteria that are closely related infect different niches within the same host (Figure 1). For H. pylori, normal stomach mucosal tissue is mostly glycosylated with fucosylated structures such as Lewis B and is rich in lactoceramides, while being poor in sialylated glycoconjugates. The inflamed stomach, however, produces a highly sialylated mucosal layer therefore requiring $H$. pylori to recognize sialic acid in order to maintain chronic infections (Mahdavi et al., 2002). In contrast, for C. jejuni, the initial interactions in the intestinal tissues are with proteins such as MUC1, a highly sialylated and mannosylated structure. MUC1, a cell surface bound mucin, is a decoy receptor and is released from by the intestinal epithelium into the intestinal lumen when recognized by pathogens such as C. jejuni (McAuley et al., 2007; Linden et al., 2008). Due to this the continued binding of sialic acid by $C$. jejuni, once it has begun to adjust to the host environment, would be detrimental to long term infection. The crypts of the intestinal epithelium, C. jejuni's favored niche, are rich in a gel forming mucin MUC2, a protein with significantly less sialylation than MUC1. It is here in the crypts and at the epithelial surface, away from the lumen of the intestinal tract, that $C$. jejuni would gain advantage from recognizing fucosylated and terminal galactose structures as these become the predominant structures presented by the host (Day et al., 2009).

We have also shown that adherence to cultured Caco- 2 colonic epithelial cells could be significantly reduced when $C$. jejuni binding was competed against commercially available lectins, particularly those lectins with specificity for fucose and galactose (Day et al., 2009). Almost complete inhibition of adherence to Caco-2 cells was observed when cells were pre-incubated with the galactose recognizing lectin ECA (90\% inhibition of $11168-\mathrm{O}$ grown at $42^{\circ} \mathrm{C}$ ) or the fucose binding lectin UEA-1 (95-100\% inhibition of 11168-O; Day et al., 2009). Previous studies had already identified fucosylated glycans as crucial for interactions between
C. jejuni and host tissues (Morrow et al., 2005). These studies identified that fucosylated glycoproteins, present in human breast milk, could inhibit C. jejuni colonization, with Lewis B, and 2fucosylactose being identified as the key glycans recognized by $C$. jejuni (Morrow et al., 2005). These data together with our findings highlight the crucial nature of glycan based interactions for C. jejuni infection and colonization. It is therefore crucial that the factors involved in the recognition of host glycans be elucidated.

Intriguingly, $C$. jejuni does not contain any orthologs or homologs of the H. pylori SabA, BabA, Hbp HapA, or HP0721 lectins but does contain a protein with $65 \%$ identity to the sialic acid binding lectin HP-NAP (Parkhill et al., 2000; Gundogdu et al., 2007). Whether this HP-NAP homolog can act as an adhesin for C. jejuni is yet to be determined. To date, bioinformatic analyses have failed to identify definite glycan recognizing proteins and the mechanisms for the recognition of such a diverse range of glycans by $C$. jejuni remains a mystery. Mechanisms, other than protein based adherence, however, may be involved in this diverse glycan recognition. One such mechanism may rely on the C. jejuni's own surface exposed glycosylation such as LOS and capsular polysaccharides (CPS) which have both been linked to adherence of C. jejuni to host cells in culture, indicating a potential role for the bacterially produced glycans in the recognition of host tissues (Fry et al., 2000).

\section{CAMPYLOBACTER JEJUNI SURFACE GLYCOSYLATION}

Campylobacter jejuni was the first bacteria to be identified with the mechanism for $\mathrm{N}$-linked protein glycosylation and displays a range of glycosylated structures including $\mathrm{N}$ - and O-linked glycoproteins, LOS, and CPS (Karlyshev et al., 2005b). N-linked glycans can either be surface expressed structures or produced as a free oligosaccharide (Nothaft and Szymanski, 2010; Nothaft et al., 2010). Interestingly, though the flippase involved in the attachment of the $\mathrm{N}$-linked glycan to the surface is non-specific for the structure it translocates, $C$. jejuni produces an N-linked glycan structure that is highly conserved between strains (Young et al., 2002; Linton et al., 2005; Kelly et al., 2006). O-linked glycosylation in C. jejuni is exclusively found on the flagella and is critical for correct flagella assembly, motility, and flagella dependent adherence (Thibault et al., 2001; Szymanski et al., 2003; Logan et al., 2009). While there is some diversity in the expression of the O-linked glycans produced by different strains, the greatest diversity in glycosylation structures is observed for both CPS and LOS (Moran, 1997; Moran and Penner, 1999; Karlyshev et al., 2005a,b). CPS and LOS are also one of the most immunogenic of the surface glycosylations presented by C. jejuni explaining much of the strain specific immunity commonly seen with $C$. jejuni infections (Karlyshev et al., 2005b; Perera et al., 2007; Monteiro et al., 2009). The glycan structures of the CPS are variable between strains with the heat stable antigen typing, which types the combination of CPS and LOS present, being one of the ways in which $C$. jejuni strains can be differentiated from one another (Moran and Penner, 1999). However, while structure of CPS is differential between strains and can be heterogenous in the same strain, the structure of the outer core sugars of the LOS is almost always heterogeneous within a population of C. jejuni of the same strain (Linton et al., 2000a; Guerry et al., 2002; Semchenko et al., 2010). 


\section{LIPOOLIGOSACCHARIDE BIOSYNTHESIS CLUSTERS: LINK TO GUILLAIN-BARRÉ SYNDROME}

Lipooligosaccharide are glycolipids expressed by Gram negative bacteria as part of their outer membrane. LOS is comprised of a lipid A molecule attached to a polysaccharide chain with a conserved inner core and a variable outer core. The outer core may be present as a single copy (LOS) as seen by the pathogen Neisseria meningitides or may repeat multiple times to produce lipopolysaccharide (LPS) as seen in a range of bacteria including E. coli (Zhu et al., 2002; Wang and Quinn, 2010). The LOS of C. jejuni has been widely studied and has been hypothesized to play important roles in cellular survival, host evasion, and adherence to host tissues (Karlyshev et al., 2005b). Strain to strain variation observed for LOS structures presented by $C$. jejuni has been linked to the diversity of the genes present within the LOS biosynthesis clusters (Gilbert et al., 2002). More then 20 different LOS biosynthesis clusters have been identified in $C$. jejuni strains, including the five capable of sialylation and ganglioside mimicry (A, B, C, M, and R; Gilbert et al., 2008). One biosynthesis cluster type, type E (C. jejuni 81116), also produces a larger LPS molecule rather than an LOS (Gilbert et al., 2008). The type $\mathrm{E}$ biosynthesis cluster is divergent from those seen in other $C$. jejuni precursor clusters contributing to the generation of a differential core oligosaccharide (Gilbert et al., 2008).

To date, the five clusters with mechanisms for ganglioside molecular mimicry (A, B, C, M, and R), have been the major focus of research into the C. jejuni LOS (Karlyshev et al., 2005b; Yuki, 2007; Gilbert et al., 2008). The most commonly used model strains for analyzing C. jejuni NCTC11168 and 81-176 both have clusters capable of ganglioside molecular mimicry, falling into cluster types $\mathrm{C}$ and $\mathrm{B}$, respectively. There appears to be a bias in research toward sialylated LOS structures of $C$. jejuni. This is mostly due to the focus on the strains producing LOS with ganglioside mimicry due to their clinical significance in the induction of GuillainBarré syndrome (GBS; Yuki, 1997). For example, recent studies have demonstrated that $C$. jejuni with sialylated LOS binds a sialoadhesin, which in turn leads to a cascade of events that result in production of cross-reactive antibodies (Louwen et al., 2008; Mortensen et al., 2009; Heikema et al., 2010; Kuijf et al., 2010).

Campylobacter jejuni infection is usually self-limiting, but in rare cases the infection precludes a debilitating, polyneuropathic disorders GBS, or the oculomotor variant Fisher syndrome (FS; Godschalk et al., 2007). C. jejuni is the most common antecedent infection in these neuropathies and expression of LOS mimicking host gangliosides is considered necessary for the neuropathy development since such mimicry can induce the production of self-reactive antibodies (Godschalk et al., 2007). Gangliosides are glycosphingolipids occurring in high concentration in the peripheral nervous system, particularly in the nerve axon but are also present on the epithelial cells of the entire gut, stomach through to the colon (Nachamkin et al., 1998; Ang et al., 2004). The production of self-reactive antibodies against these glycolipids plays a central role in GBS and FS development (Yuki, 1997). C. jejuni has been shown to produce ganglioside mimicries, including those implicated in $\mathrm{GBS}\left(\mathrm{GM}_{1}, \mathrm{GM}_{1 \mathrm{~b}}, \mathrm{GD}_{1 \mathrm{a}}, \mathrm{GalNAc}-\mathrm{GD}_{1 \mathrm{a}}\right)$ and FS (GQ $\mathrm{G}_{1 \mathrm{~b}}, \mathrm{GT}_{\mathrm{la}}$; Yuki, 1997; Ang et al., 2004). Supporting a pathogenic role of C. jejuni in GBS, C. jejuni LOS-induced anti-GM1 ganglioside antibodies react at the nodes of Ranvier, where the axon is exposed in the nerve fiber (Moran et al., 2005), resembling the pathology observed in GBS patients, and inoculation of $C$. jejuni $\mathrm{GM}_{1}$-mimicking LOS has been reported to induce GBS-like symptoms in a rabbit model (Yuki et al., 2004). Although mimicry of gangliosides by C. jejuni LOS has been extensively studied structurally over the last two decades, it is important to note that these characterization studies were performed on strains grown almost exclusively at $37^{\circ} \mathrm{C}$. The research focus on the growth temperature of $37^{\circ} \mathrm{C}$ is completely at odds with the lifestyle $C$. jejuni which colonizes a variety of niches whose temperatures range from 25 to $42^{\circ} \mathrm{C}$ (Blaser, 1997), and with previous studies that have highlighted the role the growth temperature plays in gene regulation, expression, enzymatic function, protein folding, and general bacterial metabolism (Stintzi, 2003; Semchenko et al., 2010). All this taken together indicates that there is a need to investigate LOS genetic and structural diversity at temperatures which represent different hosts.

\section{LOS STRUCTURE AND HETEROGENEITY: HOW C. JEJUNI'S ENVIRONMENT CAN EFFECT STRUCTURE}

Campylobacter jejuni exists in wide range of temperatures and atmospheric conditions, from normal atmospheric temperatures, and oxygen levels, when in the environment, to $42^{\circ} \mathrm{C}$ and virtually anaerobic in parts of the avian gut. While $C$. jejuni is thought not to replicate when in the environment, it is capable of growth at temperatures ranging from 30 to $47^{\circ} \mathrm{C}$ and therefore is capable of growth at the body temperatures of mammalian and avian hosts, 37 and $42^{\circ} \mathrm{C}$, respectively (Blaser, 1997). It is not surprising that gene expression changes have been noted under these varying conditions including the regulation of genes found in LOS biosynthesis clusters (Stintzi, 2003). Recently, however, we have shown that gene expression may not be the only change that affects the overall LOS structure under varying conditions (Semchenko et al., 2010).

Different temperature environments may trigger events to accommodate the colonization, commensalism, pathogenesis, or dormancy of this bacterium. The lack of knowledge of the structure of C. jejuni LOS at $42^{\circ} \mathrm{C}$ compared to $37^{\circ} \mathrm{C}$ prompted us to examine the effect of incubation temperature on the phenotypic variation of LOS, including the mimicry of gangliosides, in $C$. jejuni strain NCTC11168. Variation in LOS structure was assessed by electrophoretic analysis and immunoblotting and confirmed by nuclear magnetic resonance (NMR) spectroscopy. Carbohydrate epitopes produced were assessed for ganglioside mimicry using various anti-ganglioside ligands (i.e., antibodies, lectins, and cholera toxin) as probes. In addition, LOS structural variation at these two incubation temperatures was examined in minimally subcultured C. jejuni isolates from humans and chickens. Importantly, notable differences were observed in the relative production by C. jejuni 11168 of varying size and ganglioside mimicries at 37 and $42^{\circ} \mathrm{C}$. At $37^{\circ} \mathrm{C}$, the LOS of C. jejuni strain 11168 was observed to be relatively homogenous, with $90 \%$ of the total LOS produced with structure mimicking GM1 (Semchenko et al., 2010). This changed dramatically at $42^{\circ} \mathrm{C}$, with diversity in the LOS increasing, including an increased expression of asialo-GM1) resulting in 
just over $50 \%$ of the LOS structure present at $42^{\circ} \mathrm{C}$ consisting of the GM1-like structure seen to be dominant at $37^{\circ} \mathrm{C}$ (Semchenko et al., 2010). The diversity in the structures seen at different temperatures may be the result of changes in gene expression, enzymatic functionality, and metabolic changes, all potentially contributing. In addition, the C. jejuni LOS biosynthesis clusters are also known to contain several phase variable genes which are subject to change. $C$. jejuni strains have also been observed to functionally alter some of their glycosyltransferases through non-phase variable alterations, such as point mutations, on/off status, and substrate-acceptor specificity (Linton et al., 2000a; Guerry et al., 2002; Semchenko et al., 2010).

The LOS structures of two strains of C. jejuni, NCTC11168 and 81-176, have been extensively studied to identify the different forms of gangliosides they can mimic and what genes/conditions alter the host cell mimicry they can present (Linton et al., 2000a; Guerry et al., 2002; Semchenko et al., 2010). C. jejuni NCTC11168 has class $\mathrm{C}$ biosynthesis cluster that typically produces LOS that mimics monosialylated gangliosides such as GM1-2 as a result of differential expression of galactosyltransferase - wlaN (Linton et al., 2000a; St. Michael et al., 2002; Semchenko et al., 2010, 2011). However, recently we have demonstrated that cluster $\mathrm{C}$ strains are also capable of regulating LOS sialylation via inactivation of the sialytransferase - cst with a transient mutation in the gene reading frame (Semchenko et al., 2010, 2011). Thus cluster C strains are capable of producing LOS structures with GM1, GM2, asialoGM1, and asialo-GM2 like mimicries, and depending on what type of cst gene (cstII or cstIII) they encode, they may also mono- or di-sialylate the LOS, bringing the total of potential structures to six from a single cluster type.

Campylobacter jejuni 81-176 has a class B biosynthesis cluster that, depending on the sialytransferase expressed, encodes the machinery to produce ganglioside mimic ranging from monosialylated GM2-3 to GD structures with GM2 and GM3 being the most commonly observed in the wild-type organism (Guerry et al., 2002). The genes most responsible for this mimicry are the genes coding for biosynthesis of sialic acid from $N$-acetylmannose to CMP-Neu5Ac (NeuA, NeuB, NeuC) and a sialic acid transferase (CstII or CstIII; Linton et al., 2000b; Gilbert et al., 2002, 2008). Interestingly, the class C LOS biosynthesis cluster of $C$. jejuni has a gene that encodes both NeuA (CMP-Neu5Ac synthase) and CgtA (GalNAc transferase) in a single enzyme (Gilbert et al., 2008). We hypothesize that the presence of this enzyme is the reason for some of the heterogeneity observed between different growth conditions. Only half the LOS of C. jejuni 11168 is sialylated when grown under conditions mimicking the commensal avian host but nearly $90 \%$ being sialylated at conditions mimicking mammalian hosts (Semchenko et al., 2010, 2011). This change in sialylation is not necessarily related to a change in the on/off expression status of cst, but, we believe, can be due to the differences in optimal or suboptimal enzymatic function under different environmental stresses. This mechanism allows production of LOS that mimics various gangliosides in a host dependant manner, without needing to phase vary its sialic acid biosynthesis genes (Semchenko et al., 2010, under revision). This allows the entire population to produce different range of molecules, rather then only a few cells that underwent a phase change, thus avoiding a population bottleneck and increasing the fitness of the infecting population.

In other pathogens variability of surface antigens such as LOS and protein glycosylation enable the pathogen to better evade the immune system (Jarvis and Vedros, 1987; Platt et al., 1994; Khatua et al., 2010). This works by the host selecting or enriching for the bacteria that present surface antigens that have not yet triggered an immune response (Figure $\mathbf{2 A}$ ). However, the way that LOS is synthesized in C. jejuni, may result in heterogenous surface expression of LOS structures on a single bacteria. Single colonies of $C$. jejuni NCTC11168, when screened with anti-GM $\mathrm{G}_{1}$ antibodies or cholera toxin, show uniform reactivity with the lectin/antibody, suggesting uniform expression of the surface $\mathrm{GM}_{1}$ epitope. However, when the LOS preparations from these colonies are separated electrophoretically, and then screened with anti-GM $\mathrm{GM}_{1}$ antibodies or cholera toxin, there is heterogeneity in each sample with both $\mathrm{GM}_{1}$ and asialo-GM $\mathrm{GM}_{1}$ present (Semchenko et al., 2010, 2011). We therefore propose that $C$. jejuni are likely to be heterogenous for LOS presentation on a single bacteria limiting host based selection of successful C. jejuni clones and potentially this heterogenic LOS

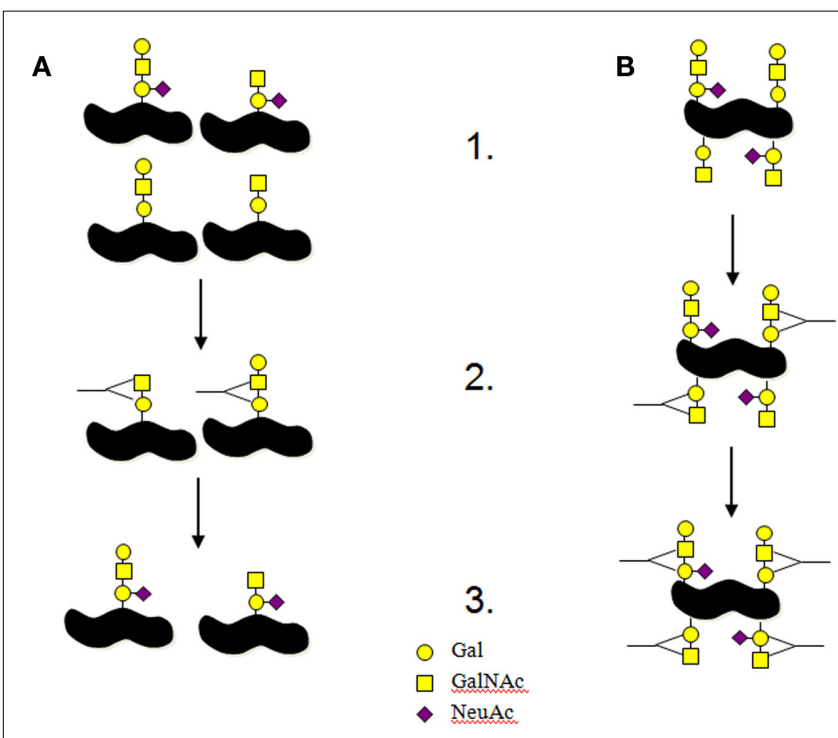

FIGURE 2 | Interactions between bacterial antigens and host immune system. (A) This is the normal process for the interactions between host immune systems and pathogenic bacteria. (1) A portion of the bacterial colony produces different antigens on their surfaces. (2) The immune system targets the surface structures that are presented in a part of the population resulting in other antigens being selected to remain. In this example antibodies are produced to non-sialylated LOS structures. (3) The antigens not targeted by the immune system are allowed to expand causing the infection. (B) This is the model we think is happening for $C$. jejuni LOS. (1) Each bacterium produces all potential surface antigens in different concentrations. (2) The immune system targets the surface structures that are presented in the population resulting antibody production against a portion of the surface antigens. In this example antibodies are produced to non-sialylated LOS structures. (3) The antigens not targeted by antibodies are still present on the targeted bacteria resulting in the increased likelihood of producing antibodies to those other LOS antigens. If the host has a defect in the mechanisms of ensuring antibodies are not produced against self then $C$. jejuni producing multiple antigens per bacteria offers a greater risk for the production of anti-self antibodies. 
may actually contribute to the production of anti-self antibodies seen in GBS/FS (Figure 2B).

\section{CONCLUSION}

Glycosylation of both the host surface and the bacterial surface is crucial for the host-pathogen interactions observed

\section{REFERENCES}

Ang, C. W., Jacobs, B. C., and Laman, J. D. (2004). The Guillain-Barre syndrome: a true case of molecular mimicry. Trends Immunol. 25, 61-66.

Aprikian, P., Tchesnokova, V., Kidd, B., Yakovenko, O., Yarov-Yarovoy, V., Trinchina, E., Vogel, V., Thomas, W., and Sokurenko, E. (2007). Interdomain interaction in the $\mathrm{FimH}$ adhesin of Escherichia coli regulates the affinity to mannose. J. Biol. Chem. 282, 23437-23446.

Bennett, H. J., and Roberts, I. S. (2005). Identification of a new sialic acid-binding protein in Helicobacter pylori. FEMS Immunol. Med. Microbiol. 44, 163-169.

Blaser, M. J. (1997). Epidemiological and clinical features of Campylobacter jejuni infection. J. Infect. Dis. 176, 103-105.

Cooksley, C., Jenks, P. J., Green, A., Cockayne, A., Logan, R. P., and Hardie, K. R. (2003). NapA protects Helicobacter pylori from oxidative stress damage, and its production is influenced by the ferric uptake regulator. J. Med. Microbiol. 52, 461-469.

Day, C. J., Tiralongo, J., Hartnell, R. D., Logue, C. A., Wilson, J. C., Von Itzstein, M., and Korolik, V. (2009). Differential carbohydrate recognition by Campylobacter jejuni strain 11168: influences of temperature and growth conditions. PLoS ONE 4, e4927. doi:10.1371/journal.pone.0004927

Fry, B. N., Feng, S., Chen, Y. Y., Newell, D. G., Coloe, P. J., and Korolik, V. (2000). The galE gene of Campylobacter jejuni is involved in lipopolysaccharide synthesis and virulence. Infect. Immun. 68, 2594-2601.

Gilbert, M., Karwaski, M. F., Bernatchez, S., Young, N. M., Taboada, E., Michniewicz, J., Cunningham, A. M., and Wakarchuk, W. W. (2002). The genetic bases for the variation in the lipo-oligosaccharide of the mucosal pathogen, Campylobacter jejuni. Biosynthesis of sialylated ganglioside mimics in the core oligosaccharide. J. Biol. Chem. 277, 327-337.

Gilbert, M., Parker, C. T., and Moran, A. P. (2008). Campylobacter jejuni
Lipooligosaccharides: Structures and Biosynthesis. Washington, DC: ASM Press.

Gilboa-Garber, N., Sudakevitz, D., Sheff, M., Sela, R., and Levene, C. (1994). PA-I and PA-II lectin interactions with the $\mathrm{ABO}(\mathrm{H})$ and $\mathrm{P}$ blood group glycosphingolipid antigens may contribute to the broad spectrum adherence of Pseudomonas aeruginosa to human tissues in secondary infections. Glycoconj. J. 11, 414-417.

Godschalk, P. C., Kuijf, M. L., Li, J., St. Michael, F., Ang, C. W., Jacobs, B. C., Karwaski, M. F., Brochu, D., Moterassed, A., Endtz, H. P., Van Belkum, A., and Gilbert, M. (2007). Structural characterization of Campylobacter jejuni lipooligosaccharide outer cores associated with Guillain-Barre and Miller Fisher syndromes. Infect. Immun. 75, 1245-1254.

Guerry, P., Szymanski, C. M., Prendergast, M. M., Hickey, T. E., Ewing, C. P., Pattarini, D. L., and Moran, A. P. (2002). Phase variation of Campylobacter jejuni 81-176 lipooligosaccharide affects ganglioside mimicry and invasiveness in vitro. Infect. Immun. 70, 787-793.

Gundogdu, O., Bentley, S. D., Holden, M. T., Parkhill, J., Dorrell, N., and Wren, B. W. (2007). Re-annotation and re-analysis of the Campylobacter jejuni NCTC11168 genome sequence. BMC Genomics 8, 162. doi:10.1186/1471-2164-8-162

Heikema, A. P., Bergman, M. P., Richards, H., Crocker, P. R., Gilbert, M., Samsom, J. N., Van Wamel, W. J., Endtz, H. P., and Van Belkum, A. (2010). Characterization of the specific interaction between sialoadhesin and sialylated Campylobacter jejuni lipooligosaccharides. Infect. Immun. 78, 3237-3246.

Hugdahl, M. B., Beery, J. T., and Doyle, M. P. (1988). Chemotactic behavior of Campylobacter jejuni. Infect. Immun. 56, 1560-1566.

Hynes, S. O., Teneberg, S., Roche, N., and Wadstrom, T. (2003). Glycoconjugate binding of gastric and enterohepatic Helicobacter spp. Infect. Immun. 71, 2976-2980.

for C. jejuni. A better understanding of the factors involved in glycan expression and recognition by both the bacteria and the host may offer new insights into the mechanisms involved in C. jejuni commensal colonization, pathogenic infection, and the induction of polyneuropathic disorders GBS and FS.

Ilver, D., Arnqvist, A., Ogren, J., Frick, I. M., Kersulyte, D., Incecik, E. T. Berg, D. E., Covacci, A., Engstrand, L., and Boren, T. (1998). Helicobacter pylori adhesin binding fucosylated histo-blood group antigens revealed by retagging. Science 279 , 373-377.

Jarvis, G. A., and Vedros, N. A. (1987). Sialic acid of group B Neisseria meningitidis regulates alternative complement pathway activation. Infect. Immun. 55, 174-180.

Karlyshev, A. V., Champion, O. L., Churcher, C., Brisson, J. R., Jarrell, H. C., Gilbert, M., Brochu, D. St. Michael, F., Li, J., Wakarchuk, W. W., Goodhead, I., Sanders, M., Stevens, K., White, B., Parkhill, J. Wren, B. W., and Szymanski, C. M. (2005a). Analysis of Campylobacter jejuni capsular loci reveals multiple mechanisms for the generation of structural diversity and the ability to form complex heptoses. Mol. Microbiol. 55, 90-103.

Karlyshev, A. V., Ketley, J. M., and Wren, B. W. (2005b). The Campylobacter jejuni glycome. FEMS Microbiol. Rev. 29, 377-390.

Kelly, J., Jarrell, H., Millar, L., Tessier, L., Fiori, L. M., Lau, P. C., Allan, B., and Szymanski, C. M. (2006). Biosynthesis of the N-linked glycan in Campylobacter jejuni and addition onto protein through block transfer. $J$. Bacteriol. 188, 2427-2434.

Khatua, B., Ghoshal, A., Bhattacharya, K., Mandal, C., Saha, B., and Crocker, P. R. (2010). Sialic acids acquired by Pseudomonas aeruginosa are involved in reduced complement deposition and siglec mediated host-cell recognition. FEBS Lett. 584, 555-561.

Klemm, P., Schembri, M., and Hasty, D. L. (1996). The FimH protein of type 1 fimbriae: an adaptable adhesin. Adv. Exp. Med. Biol. 408, 193-195.

Kuijf, M. L., Samsom, J. N., Van Rijs, W., Bax, M., Huizinga, R., Heikema, A. P., Van Doorn, P. A., Van Belkum, A., Van Kooyk, Y., Burgers, P. C., Luider, T. M., Endtz, H. P., Nieuwenhuis, E. E., and Jacobs, B. C. (2010). TLR4mediated sensing of Campylobacter jejuni by dendritic cells is determined by sialylation. J. Immunol. $185,748-755$.
Lehmann, F., Tiralongo, E., and Tiralongo, J. (2006). Sialic acid-specific lectins: occurrence, specificity and function. Cell. Mol. Life Sci. 63, 1331-1354.

Linden, S. K., Sutton, P., Karlsson, N. G., Korolik, V., and Mcguckin, M. A. (2008). Mucins in the mucosal barrier to infection. Mucosal Immunol. 1, 183-197.

Linton, D., Dorrell, N., Hitchen, P. G., Amber, S., Karlyshev, A. V., Morris, H. R., Dell, A., Valvano, M. A., Aebi, M., and Wren, B. W. (2005). Functional analysis of the Campylobacter jejuni $\mathrm{N}$-linked protein glycosylation pathway. Mol. Microbiol. 55, 1695-1703.

Linton, D., Gilbert, M., Hitchen, P. G., Dell, A., Morris, H. R., Wakarchuk, W. W., Gregson, N. A., and Wren, B. W. (2000a). Phase variation of a beta-1,3 galactosyltransferase involved in generation of the ganglioside GM1-like lipooligosaccharide of Campylobacter jejuni. Mol. Microbiol. 37, 501-514.

Linton, D., Karlyshev, A. V., Hitchen, P. G., Morris, H. R., Dell, A., Gregson, N. A., and Wren, B. W. (2000b). Multiple $N$-acetyl neuraminic acid synthetase (neuB) genes in Campylobacter jejuni: identification and characterization of the gene involved in sialylation of lipo-oligosaccharide. Mol. Microbiol. 35, 1120-1134.

Lloyd, D. H., Viac, J., Werling, D., Reme, C. A., and Gatto, H. (2007) Role of sugars in surface microbehost interactions and immune reaction modulation. Vet. Dermatol. 18, 197-204.

Logan, S. M., Hui, J. P., Vinogradov, E., Aubry, A. J., Melanson, J. E., Kelly, J. F., Nothaft, H., and Soo, E. C. (2009). Identification of novel carbohydrate modifications on Campylobacter jejuni 11168 flagellin using metabolomics-based approaches. FEBS J. 276, 1014-1023.

Louwen, R., Heikema, A., Van Belkum, A., Ott, A., Gilbert, M., Ang, W., Endtz, H. P., Bergman, M. P., and Nieuwenhuis, E. E. (2008). The sialylated lipooligosaccharide outer core in Campylobacter jejuni is an important determinant for epithelial cell invasion. Infect. Immun. 76, 4431-4438. 
Mahdavi, J., Sonden, B., Hurtig, M., Olfat, F. O., Forsberg, L., Roche, N., Angstrom, J., Larsson, T., Teneberg, S., Karlsson, K. A., Altraja, S., Wadstrom, T., Kersulyte, D., Berg, D. E., Dubois, A., Petersson, C., Magnusson, K. E., Norberg, T., Lindh, F., Lundskog, B. B., Arnqvist, A., Hammarstrom, L., and Boren, T. (2002). Helicobacter pylori SabA adhesin in persistent infection and chronic inflammation. Science 297, 573-578.

McAuley, J. L., Linden, S. K., Png, C. W., King, R. M., Pennington, H. L., Gendler, S. J., Florin, T. H., Hill, G. R., Korolik, V., and McGuckin, M. A. (2007). MUC1 cell surface mucin is a critical element of the mucosal barrier to infection. J. Clin. Invest. 117, 2313-2324.

Monteiro, M. A., Baqar, S., Hall, E. R., Chen, Y. H., Porter, C. K., Bentzel, D. E., Applebee, L., and Guerry, P. (2009). Capsule polysaccharide conjugate vaccine against diarrheal disease caused by Campylobacter jejuni. Infect. Immun. 77, 1128-1136.

Moran, A. P. (1997). Structure and conserved characteristics of Campylobacter jejuni lipopolysaccharides. J. Infect. Dis. 176(Suppl. 2), S115S121.

Moran, A. P., Annuk, H., and Prendergast, M. M. (2005). Antibodies induced by ganglioside-mimicking Campylobacter jejuni lipooligosaccharides recognise epitopes at the nodes of Ranvier. J. Neuroimmunol. 165, 179-185.

Moran, A. P., and Penner, J. L. (1999). Serotyping of Campylobacter jejuni based on heat-stable antigens: relevance, molecular basis and implications in pathogenesis. J. Appl. Microbiol. 86, 361-377.

Morrow, A. L., Ruiz-Palacios, G. M., Jiang, X., and Newburg, D. S. (2005). Human-milk glycans that inhibit pathogen binding protect breast-feeding infants against infectious diarrhea. J. Nutr. 135, 1304-1307.

Mortensen, N. P., Kuijf, M. L., Ang, C. W., Schiellerup, P., Krogfelt, K. A., Jacobs, B. C., Van Belkum, A., Endtz, H. P., and Bergman, M. P. (2009). Sialylation of Campylobacter jejuni lipo-oligosaccharides is associated with severe gastro-enteritis and reactive arthritis. Microbes Infect. 11, 988-994.

Nachamkin, I., Allos, B. M., and Ho, T. (1998). Campylobacter species and Guillain-Barre syndrome. Clin. Microbiol. Rev. 11, 555-567.
Nothaft, H., Liu, X., Li, J., and Szymanski, C. M. (2010). Campylobacter jejuni free oligosaccharides: function and fate. Virulence 1 546-550.

Nothaft, H., and Szymanski, C. M. (2010). Protein glycosylation in bacteria: sweeter than ever. Nat. Rev. Microbiol. 8, 765-778.

Parkhill, J., Wren, B. W., Mungall, K., Ketley, J. M., Churcher, C., Basham, D., Chillingworth, T., Davies, R. M., Feltwell, T., Holroyd, S., Jagels, K., Karlyshev, A. V., Moule, S., Pallen, M. J., Penn, C. W., Quail, M. A., Rajandream, M. A., Rutherford, K. M., Van Vliet, A. H., Whitehead, S., and Barrell, B. G. (2000). The genome sequence of the food-borne pathogen Campylobacter jejuni reveals hypervariable sequences. Nature 403, 665-668.

Perera, V. N., Nachamkin, I., Ung, H., Patterson, J. H., Mcconville, M. J., Coloe, P. J., and Fry, B. N. (2007). Molecular mimicry in Campylobacter jejuni: role of the lipo-oligosaccharide core oligosaccharide in inducing anti-ganglioside antibodies. FEMS Immunol. Med. Microbiol. 50, 27-36.

Platt, M. W., Correa, N. Jr., and Mold, C. (1994). Growth of group B streptococci in human serum leads to increased cell surface sialic acid and decreased activation of the alternative complement pathway. Can. J. Microbiol. 40, 99-105.

Pouttu, R., Puustinen, T., Virkola, R., Hacker, J., Klemm, P., and Korhonen, T. K. (1999). Amino acid residue Ala-62 in the FimH fimbrial adhesin is critical for the adhesiveness of meningitis-associated Escherichia coli to collagens. Mol. Microbiol. 31, 1747-1757.

Rosen, D. A., Pinkner, J. S., Walker, J. N., Elam, J. S., Jones, J. M. and Hultgren, S. J. (2008). Molecular variations in Klebsiella pneumoniae and Escherichia coli FimH affect function and pathogenesis in the urinary tract. Infect. Immun. 76, 3346-3356.

Scharfman, A., Degroote, S., Beau, J., Lamblin, G., Roussel, P., and Mazurier, J. (1999). Pseudomonas aeruginosa binds to neoglycoconjugates bearing mucin carbohydrate determinants and predominantly to sialyl-Lewis $\mathrm{x}$ conjugates. Glycobiology 9, 757-764.

Semchenko, E. A., Day, C. J., Wilson, J. C., Grice, I. D., Moran, A. P., and
Korolik, V. (2010). Temperaturedependent phenotypic variation of Campylobacter jejuni lipooligosaccharides. BMC Microbiol. 10, 305. doi: 10.1186/1471-2180-10305

Semchenko, E. A., Moutin, M., Korolik, V., Tiralongo, T., and Day, C. J. (2011). Lectin array analysis of purified lipooligosaccharide: a method for the determination of molecular mimicry. J. Glycom. Lipidom. 1, $1-5$.

Sepehri, S., Kotlowski, R., Bernstein, C. N., and Krause, D. O. (2009). Phylogenetic analysis of inflammatory bowel disease associated Escherichio coli and the fimH virulence determinant. Inflamm. Bowel Dis. 15, 1737-1745.

Sharon, N. (2008). Lectins: past, present and future. Biochem. Soc. Trans. 36, 1457-1460.

Sokurenko, E. V., Chesnokova, V. Dykhuizen, D. E., Ofek, I., Wu, X. R., Krogfelt, K. A., Struve, C., Schembri, M. A., and Hasty, D. L. (1998). Pathogenic adaptation of Escherichia coli by natural variation of the FimH adhesin. Proc. Natl. Acad. Sci. U.S.A. 95, 8922-8926.

Sokurenko, E. V., Courtney, H. S. Ohman, D. E., Klemm, P., and Hasty, D. L. (1994). FimH family of type 1 fimbrial adhesins: functional heterogeneity due to minor sequence variations among fimH genes. J. Bacteriol. 176, 748-755.

St. Michael, F., Szymanski, C. M., Li, J., Chan, K. H., Khieu, N. H., Larocque, S., Wakarchuk, W. W., Brisson, J. R., and Monteiro, M. A. (2002). The structures of the lipooligosaccharide and capsule polysaccharide of Campylobacter jejuni genome sequenced strain NCTC 11168. Eur. J. Biochem. 269, 5119-5136.

Stahl, M., Friis, L. M., Nothaft, H., Liu, X., Li, J., Szymanski, C. M., and Stintzi, A. (2011). L-Fucose utilization provides Campylobacter jejuni with a competitive advantage. Proc. Natl. Acad. Sci. U.S.A. 108 7194-7199.

Stintzi, A. (2003). Gene expression profile of Campylobacter jejuni in response to growth temperature variation. J. Bacteriol. 185, 2009-2016.

Szymanski, C. M., Logan, S. M., Linton, D., and Wren, B. W. (2003). Campylobacter - a tale of two protein glycosylation systems. Trends Microbiol. 11, 233-238.

Thibault, P., Logan, S. M., Kelly, J. F., Brisson, J. R., Ewing, C. P., Trust,
T. J., and Guerry, P. (2001). Identification of the carbohydrate moieties and glycosylation motifs in Campylobacter jejuni flagellin. J. Biol. Chem. 276, 34862-34870.

Thomas, W. E., Trintchina, E., Forero, M., Vogel, V., and Sokurenko, E. V. (2002). Bacterial adhesion to target cells enhanced by shear force. Cell 109, 913-923.

Tu, Q. V., Mcguckin, M. A., and Mendz, G. L. (2008). Campylobacter jejuni response to human mucin MUC2: modulation of colonization and pathogenicity determinants. J. Med. Microbiol. 57, 795-802.

Utt, M., and Wadstrom, T. (1997). Identification of heparan sulphate binding surface proteins of Helicobacter pylori: inhibition of heparan sulphate binding with sulphated carbohydrate polymers. J. Med. Microbiol. 46, 541-546.

Walz, A., Odenbreit, S., Mahdavi, J., Boren, T., and Ruhl, S. (2005). Identification and characterization of binding properties of Helicobacter pylori by glycoconjugate arrays. Glycobiology 15, 700-708.

Wang, X., and Quinn, P. J. (2010). Lipopolysaccharide: biosynthetic pathway and structure modification. Prog. Lipid Res. 49, 97-107.

Yajima, A., Urano-Tashiro, Y., Shimazu, K., Takashima, E., Takahashi, Y., and Konishi, K. (2008). Hsa, an adhesin of Streptococcus gordonii DL1, binds to alpha2-3-linked sialic acid on glycophorin A of the erythrocyte membrane. Microbiol. Immunol. 52, 69-77.

Young, N. M., Brisson, J. R., Kelly, J., Watson, D. C., Tessier, L., Lanthier, P. H., Jarrell, H. C., Cadotte, N., St. Michael, F., Aberg, E., and Szymanski, C. M. (2002). Structure of the N-linked glycan present on multiple glycoproteins in the Gram-negative bacterium, Campy lobacter jejuni. J. Biol. Chem. 277, 42530-42539.

Yuki, N. (1997). Molecular mimicry between gangliosides and lipopolysaccharides of Campylobacter jejuni isolated from patients with Guillain-Barre syndrome and Miller Fisher syndrome. J. Infect. Dis. 176(Suppl. 2), S150-S153.

Yuki, N. (2007). Campylobacter sialyltransferase gene polymorphism directs clinical features of GuillainBarre syndrome. J. Neurochem. 103(Suppl. 1), 150-158.

Yuki, N., Susuki, K., Koga, M., Nishimoto, Y., Odaka, M., Hirata, K. 
Taguchi, K., Miyatake, T., Furukawa, K., Kobata, T., and Yamada, M. (2004). Carbohydrate mimicry between human ganglioside GM1 and Campylobacter jejuni lipooligosaccharide causes GuillainBarre syndrome. Proc. Natl. Acad. Sci. U.S.A. 101, 11404-11409.

Zhu, P., Klutch, M. J., Bash, M. C., Tsang, R. S., Ng, L. K., and Tsai,
C. M. (2002). Genetic diversity of three lgt loci for biosynthesis of lipooligosaccharide (LOS) in Neisseria species. Microbiology 148, 1833-1844.

Conflict of Interest Statement: The authors declare that the research was conducted in the absence of any commercial or financial relationships that could be construed as a potential conflict of interest.

Received: 31 October 2011; accepted: 29 January 2012; published online: 14 February 2012

Citation: Day CJ, Semchenko EA and Korolik V (2012) Glycoconjugates play a key role in Campylobacter jejuni infection: interactions between host and pathogen. Front. Cell. Inf. Microbio. 2:9. doi: 10.3389/fcimb.2012.00009

Copyright (c) 2012 Day, Semchenko and Korolik. This is an open-access article distributed under the terms of the Creative Commons Attribution Non Commercial License, which permits noncommercial use, distribution, and reproduction in other forums, provided the original authors and source are credited. 